\title{
Mulheres negras e brancas e os níveis de acesso aos serviços preventivos de saúde: uma análise sobre as desigualdades
}

Black and white women and the accessibility to preventive health services: an analysis

of inequalities

Emanuelle Freitas Goes', Enilda Rosendo do Nascimento²

${ }^{1}$ Mestre em Enfermagem pela Universidade Federal da Bahia (UFBA)

- Salvador (BA), Brasil. Professora

Substituta da Escola de Enfermagem da Universidade Federal da Bahia (UFBA) -

Salvador (BA), Brasil.

emanuellegoes@gmail.com

2 Doutora em Enfermagem pela Universidade Federal do Rio de Janeiro (UFRJ) - Rio de Janeiro (RJ), Brasil.

Professora do Programa de Pós-graduação

em Enfermagem da Universidade Federal da Bahia (UFBA) - Salvador (BA), Brasil.

enildarosendo@hotmail.com
RESUMO: O racismo institucional é um fator determinante no acesso aos serviços de saúde, principalmente para as mulheres negras que sofrem com o impacto das intersecções das desigualdades de gênero e raça. O objetivo deste estudo é determinar os diferenciais das características sócio demográficas e os níveis de acesso aos serviços preventivos de mulheres na Bahia, segundo raça/cor. Os resultados revelam que, para o nível de acesso considerado bom, as mulheres brancas representam 15,4\%, enquanto as negras respondem por $7,9 \%$. O estudo demonstrou que as desigualdades raciais e o racismo institucional são uma barreira no acesso aos serviços preventivos de saúde para as mulheres negras.

PALAVRAS-CHAVE: Desigualdades em saúde; Racismo; Acesso aos serviços de saúde; Saúde da mulher

ABSTRACT: Institutional racism is a determining factor for accessing health services, mainly for those black women who suffer the impact of gender and race inequalities' intersections. This study aims to ascertain the differences among socio-demographic characteristics and access levels to preventive services for women in Bahia as for race/color. The results noted that from those entitled to the good access level $15.4 \%$ were white women while $7.9 \%$ were black ones. The study showed that racial inequalities and institutional racism are barriers against the access to preventive health services regarding black women.

KEYWORDS: Inequalities in health; Racism; Access to health services; Women's health 


\section{Introdução}

A desigualdade não decorre da diferença individual, mas do modo como as pessoas estão organizadas socialmente, em uma estrutura hierarquizada por valores simbólicos e materiais produzida pelas variadas relaçóes sociais e que tem como consequência a repartição não uniforme de todos os tipos de vantagens e desvantagens. As desigualdades sociais são, portanto, resultado de processos sociais, demográficos, econômicos, culturais e políticos desenvolvidos em contextos determinados social e historicamente.

Para Barata (2009), a discussão sobre igualdade ou desigualdade deve se situar além da simples comparação de situaçôes, atribuindo-se juízo de valor ao que é igual ou desigual. Nesse sentido, as desigualdades sociais se referem às situações injustas, porque estão associadas a características sociais que sistematicamente colocam alguns grupos em desvantagem em relação a outros quanto a oportunidades e acesso aos bens e serviços.

Nessa perspectiva, as desigualdades levam ao prejuízo do acesso à renda e aos direitos definidos como fundamentais: educação, saúde, previdência social, habitação, informação e bens culturais para grupos sociais historicamente excluídos. As desigualdades raciais no Brasil, estruturadas pelo racismo institucionalizado, impactam o acesso aos bens e serviços, tratando as pessoas de forma desigual por causa da sua raça/cor/etnia ou religião. Fruto da colonização, o racismo é reconhecido como princípio ativo desse processo, pois, desde o século $\mathrm{XVI}$, as desigualdades impostas pelo regime escravagista do Brasil se mantiveram com o desenvolvimento de um sistema fundamentado no capitalismo, que conservou o racismo como legitimador da exclusão social.

$\mathrm{O}$ racismo institucional, conceito muito utilizado no Brasil para explicar as desigualdades raciais no acesso aos serviços de saúde, é descrito como:

O fracasso das instituiçôes e organizaçóes em prover um serviço profissional e adequado às pessoas em virtude de sua cor, cultura, origem racial ou étnica. Ele se manifesta em normas, práticas e comportamentos discriminatórios adotados no cotidiano do trabalho, os quais são resultantes do preconceito racial, uma atitude que combina estereótipos racistas, falta de atenção e ignorância. Em qualquer caso, o racismo institucional sempre coloca pessoas de grupos raciais ou étnicos discriminados em situação de desvantagem no acesso a beneficios gerados pelo Estado e por demais instituiçóes e organizaçóes (CRI, 2006, p.22).

As relações raciais estão enraizadas na vida social dos grupos e classes sociais, afetando os indivíduos. Ao afetarem a capacidade de inserção das pessoas negras na sociedade brasileira, as desigualdades comprometem o projeto da construçấo de um país democrático, com oportunidades iguais para todas as pessoas, limitando, dessa forma, a capacidade de inclusão da população negra (HERINGER, 2002; IANNI, 2004).

No entanto, as pesquisas sobre as desigualdades sociais em saúde no Brasil privilegiam as análises a partir das condiçóes sócio-econômicas em seus modelos explicativos, não havendo uma produção sistemática com a dimensão étnico-racial na expressão diferenciada dos agravos à saúde, visto que a qualidade de vida dos cidadáos determina a forma de adoecer e morrer. As pesquisas mostram desigualdades no perfil de saúde entre regióes e diferentes segmentos de classe social, mas poucas associam à inserção social desqualificada/ desvalorizada da população negra na sociedade e nos indicadores de saúde (BATISTA; ESCUDER, 2005; BARATA, 1997).

A garantia do acesso universal e equitativo à saúde está diretamente associada ao acesso e utilização dos serviços que, muitas vezes, são violados por multifatores, tais como eixos estruturantes que funcionam de forma articulada, racismo, sexismo e condiçóes sócioeconômicas e culturais. $\mathrm{O}$ acesso - utilização dos serviços e insumos de saúde - é condição importante para a manutenção de bom estado de saúde ou para seu reestabelecimento, embora não seja o único fator responsável por uma vida saudável e de boa qualidade.

$\mathrm{E}$, nesse caso, as mulheres negras experimentam diferentes tipos de discriminação de raça e gênero, que, quando se interseccionam, comprometem a sua inserção na sociedade como um sujeito de direito, 
principalmente no que tange à saúde, onde as desigualdades impostas pelo racismo e sexismo diferenciam as mulheres no acesso aos serviços de saúde assim como no processo de adoecimento.

De acordo com Crenshaw (2002), a interseccionalidade é uma associação de sistemas múltiplos de subordinação, sendo descrita de várias formas, como discriminação composta, carga múltipla ou dupla ou tripla discriminação, que concentra problemas, buscando capturar as consequências estruturais de dinâmicas da interação entre dois ou mais eixos da subordinação.

As mulheres negras sofrem com o fenômeno da dupla discriminação, ou seja, estão sujeitas às múltiplas formas de discriminação social em consequência da conjugação perversa de racismo e sexismo, resultando em uma espécie de asfixia social com desdobramentos negativos em todas as dimensões da vida (CARNEIRO, 2002).

O objetivo deste artigo é analisar as desigualdades raciais e os níveis de acesso de mulheres maiores de 25 anos aos serviços preventivos de saúde na Bahia, segundo raça/cor.

\section{Material e método}

Trata-se de estudo descritivo com abordagem quantitativa. O estudo descritivo procura observar, registrar, analisar, classificar e interpretar os fatos ou fenômenos (variáveis) sem que o pesquisador interfira neles ou os manipule. Esse tipo de estudo tem como objetivo fundamental a descrição das características de determinada população ou fenômeno.

A população desta pesquisa é formada por mulheres negras, soma de pretas e pardas, e brancas com 25 anos ou mais de idade, residentes na Bahia, que responderam ao questionário da Pesquisa Nacional de Amostra por Domicílio (PNAD) em 2008.

Como fonte para o estudo, foi utilizado o Suplemento de Saúde da PNAD/IBGE, de 2008. A PNAD, realizada pelo Instituto Brasileiro de Geografia e Estatística, é um inquérito com base na população, de abrangência nacional e ocorre todos os anos. Já a Pesquisa Suplementar de Saúde, ocorre com uma periodicidade de cinco anos; iniciou-se em 1998 e foi repetida com algumas alteraçóes e inclusóes em 2003, e na terceira edição, em 2008.

A PNAD é realizada por meio de uma amostra probabilística de domicílios, obtida em três estágios de seleção: unidades primárias - municípios; unidades secundárias - setores censitários; e unidades terciárias - unidades domiciliares (domicílios particulares e unidades de habitação em domicílios coletivos). O período da coleta de dados ocorreu de 29 de agosto a 20 de setembro de 2008.

As variáveis definidas para o estudo foram categorizadas em grupos distintos: características individuais, que correspondem às seguintes variáveis raça/cor, idade e sexo; comportamento e os estilos de vida individuais relacionados à composição familiar; condiçôes econômicas, culturais e ambientais compostas por nível de instrução e renda mensal; condições de vida e de trabalho, que inclui instrução, trabalho e renda, morbidades referidas, estado de saúde e acesso a serviços preventivos de saúde.

Para uma análise sobre o acesso a serviços preventivos de saúde do câncer de mama e do colo uterino por mulheres negras e brancas, foi construído um indicador de acesso contemplando três níveis, conforme o número de exames realizados e o tipo de financiamento utilizado SUS ou Plano de Saúde. A construção do indicador de acesso tomou como base a composição das variáveis ordinais a seguir, derivadas de variáveis relacionadas ao acesso e à utilização de serviços preventivos de saúde da mulher do suplemento saúde da PNAD/IBGE (2008), sendo o indicador qualificado como bom, regular ou ruim, da seguinte forma:

Bom: a realização todos os exames preventivos (clínico das mamas, mamografia e colo de útero) e a utilização de plano de saúde ou serviços do SUS;

Regular: a realização de dois dos três exames preventivos com a utilização do plano de saúde ou serviços do SUS; e

Ruim: a realização de somente um dos três exames ou a não realização de qualquer dos três exames. 
Tabela 1. Níveis de acesso aos serviços preventivos de saúde segundo raça/cor e características sócio demográficas de mulheres com 25 anos ou mais de idade, Bahia, 2008

\begin{tabular}{|c|c|c|c|c|c|c|}
\hline \multirow{2}{*}{$\begin{array}{l}\text { CARACTERÍSTICAS } \\
\text { SÓCIO DEMOGRÁFICAS }\end{array}$} & \multicolumn{3}{|c|}{ BRANCA } & \multicolumn{3}{|c|}{ NEGRA } \\
\hline & $\begin{array}{l}\text { BOM } \\
\text { N (\%) }\end{array}$ & $\begin{array}{l}\text { REGULAR } \\
\text { N (\%) }\end{array}$ & $\begin{array}{l}\text { RUIM } \\
\text { N (\%) }\end{array}$ & $\begin{array}{l}\text { BOM } \\
\text { N (\%) }\end{array}$ & $\begin{array}{c}\text { REGULAR } \\
\text { N (\%) }\end{array}$ & $\begin{array}{l}\text { RUIM } \\
\text { N (\%) }\end{array}$ \\
\hline \multicolumn{7}{|l|}{ RAÇA/COR } \\
\hline & $331(15,4)$ & $254(11,8)$ & $1566(72,8)$ & $640(7,9)$ & $1103(13,6)$ & $6397(78,6)$ \\
\hline & \multicolumn{3}{|c|}{ Valor de $p<0,000$} & \multicolumn{3}{|c|}{ Valor de $\mathrm{p}<0,000$} \\
\hline \multicolumn{7}{|l|}{ GRUPO ETÁRIO (EM ANOS) } \\
\hline 25 a 34 & $43(7,5)$ & $69(12,1)$ & $458(80,4)$ & $93(3,8)$ & $241(9,7)$ & $2147(86,5)$ \\
\hline 35 a 49 & $122(17,5)$ & $93(13,4)$ & $481(69,1)$ & $264(9,0)$ & $473(16,2)$ & $2187(74,8)$ \\
\hline \multirow[t]{2}{*}{50 e mais } & $166(18,8)$ & $92(10,4)$ & $627(60,8)$ & $283(10,4)$ & $393(14,4)$ & $2059(75,3)$ \\
\hline & \multicolumn{3}{|c|}{ Valor de $p<0,000$} & \multicolumn{3}{|c|}{ Valor de $\mathrm{p}<0,000$} \\
\hline \multicolumn{7}{|l|}{ COMPOSIÇÃO FAMILIAR } \\
\hline Casal sem filhos & $53(15,8)$ & $36(10,8)$ & $246(73,4)$ & $92(9,7)$ & $149(14,6)$ & $777(76,3)$ \\
\hline Casal com filhos & $165(15,5)$ & $140(13,1)$ & $763(71,4)$ & $321(7,9)$ & $534(13,2)$ & $3202(78,9)$ \\
\hline Mães Solteiras & $65(13,5)$ & $54(11,2)$ & $364(75,4)$ & $143(6,2)$ & $316(14,3)$ & $1747(79,2)$ \\
\hline \multirow[t]{2}{*}{ Outros tipos de família } & $48(18,1)$ & $24(9,1)$ & $193(72,8)$ & $84(9,8)$ & $108(12,6)$ & $667(77,6)$ \\
\hline & \multicolumn{3}{|c|}{ Valor de $\mathrm{p}<0,328$} & \multicolumn{3}{|c|}{ Valor de $\mathrm{p}<0,021$} \\
\hline \multicolumn{7}{|l|}{ NÍVEL DE INSTRUÇÃO* } \\
\hline Sem instrução & $4(1,0)$ & $24(6,2)$ & $363(92,8)$ & $15(0,9)$ & $139(8,8)$ & $1426(90,6)$ \\
\hline Fundamental & $46(6,1)$ & $86(11,3)$ & $626(82,6)$ & $132(3,9)$ & $470(13,9)$ & $2771(82,6)$ \\
\hline Médio & $130(20,5)$ & $79(12,4)$ & $426(67,1)$ & $309(13,1)$ & $379(14,9)$ & $1859(73,0)$ \\
\hline \multirow[t]{2}{*}{ Superior } & $151(41,6)$ & $64(17,6)$ & $148(40,8)$ & $181(29,2)$ & $116(18,7)$ & $322(52,0)$ \\
\hline & \multicolumn{3}{|c|}{ Valor de $\mathrm{p}<0,000$} & \multicolumn{3}{|c|}{ Valor de $\mathrm{p}<0,000$} \\
\hline
\end{tabular}

Fonte: PNAD/IBGE - Pesquisa Nacional de Amostra por domicílios, 2008.

Nota: ${ }^{*}$ Nível de instrução mais elevado, inclui curso completo e incompleto.

As variáveis categóricas foram descritas por meio de distribuiçóes de frequência (uni e bi variadas). Foram realizados o teste qui-quadrado de Pearson e o exato de Fisher (quando necessário) para verificar as diferenças entre as proporçôes das características sócio-demográficas e dos níveis de acesso (bom, regular e ruim), segundo a raça/cor. Foi utilizado o programa SPSS (Statistical Package for the Social Sciences) 17.0 para a construção e armazenamento dos dados e o programa estatístico STATA v.8.0 para tratamento, construção do indicador de acesso e geração dos resultados. 
Tabela 2. Níveis de acesso aos serviços preventivos de saúde segundo raça/cor e condições de trabalho e renda de mulheres com 25 anos ou mais de idade, Bahia, 2008

\begin{tabular}{|c|c|c|c|c|c|c|}
\hline \multirow{2}{*}{$\begin{array}{l}\text { CARACTERÍSTICAS } \\
\text { DAS CONDIÇÕES DE } \\
\text { TRABALHO E RENDA }\end{array}$} & \multicolumn{3}{|c|}{ BRANCA } & \multicolumn{3}{|c|}{ NEGRA } \\
\hline & $\begin{array}{l}\text { BOM } \\
\text { N (\%) }\end{array}$ & $\begin{array}{l}\text { REGULAR } \\
\text { N (\%) }\end{array}$ & $\begin{array}{l}\text { RUIM } \\
\text { N (\%) }\end{array}$ & $\begin{array}{l}\text { BOM } \\
\text { N (\%) }\end{array}$ & $\begin{array}{l}\text { REGULAR } \\
\text { N (\%) }\end{array}$ & $\begin{array}{l}\text { RUIM } \\
\mathrm{N}(\%)\end{array}$ \\
\hline \multicolumn{7}{|c|}{ POSIÇÃO NA OCUPAÇÃO NO TRABALHO } \\
\hline $\begin{array}{l}\text { Empregada com carteira } \\
\text { assinada }\end{array}$ & $84(27,9)$ & $50(16,6)$ & $167(55,5)$ & $155(15,0)$ & $194(18,8)$ & $684(66,2)$ \\
\hline $\begin{array}{l}\text { Empregada sem carteira } \\
\text { assinada }\end{array}$ & $16(8,8)$ & $21(11,5)$ & $145(79,7)$ & $41(5,6)$ & $86(11,7)$ & $605(82,6)$ \\
\hline Funcionária pública & $47(34,6)$ & $19(14,0)$ & $70(51,5)$ & $101(27,2)$ & $53(14,3)$ & $217(58,5)$ \\
\hline $\begin{array}{l}\text { Trabalhadora } \\
\text { doméstica* }\end{array}$ & $3(2,6)$ & $10(8,7)$ & $102(88,7)$ & $12(1,3)$ & $146(15,1)$ & $808(83,6)$ \\
\hline Não remunerada & $3(2,3)$ & $13(10,1)$ & $113(87,6)$ & $5(1,5)$ & $18(5,2)$ & $321(93,3)$ \\
\hline Autônoma** & $55(13,0)$ & $51(12,1)$ & $316(74,9)$ & $92(5,5)$ & $203(12,1)$ & $1367(82,3)$ \\
\hline & \multicolumn{3}{|c|}{ Valor de $p<0,000$} & \multicolumn{3}{|c|}{ Valor de $p<0,000$} \\
\hline \multicolumn{7}{|l|}{ CONDIÇÃO DE ATIVIDADE } \\
\hline Economicamente ativa & $212(15,8)$ & $169(12,6)$ & $963(71,6)$ & $215(7,7)$ & $764(13,8)$ & $4354(78,5)$ \\
\hline \multirow[t]{2}{*}{$\begin{array}{l}\text { Não economicamente } \\
\text { ativa }\end{array}$} & $119(14,7)$ & $85(10,5)$ & $603(74,7)$ & $215(8,3)$ & $343(13,2)$ & $2039(78,5)$ \\
\hline & \multicolumn{3}{|c|}{ Valor de $p<0,249$} & \multicolumn{3}{|c|}{ Valor de $\mathrm{p}<0,529$} \\
\hline \multicolumn{7}{|c|}{ RENDA PESSOAL (EM SALÁRIOS MÍNIMOS) *** } \\
\hline Inferior a 1 & $19(3,9)$ & $48(9,9)$ & $419(86,2)$ & $46(2,1)$ & $241(10,7)$ & $1968(87,3)$ \\
\hline 1 até 3 & $81(17,4)$ & $55(11,8)$ & $330(70,8)$ & $206(10,5)$ & $336(17,2)$ & $1413(72,3)$ \\
\hline 3 até 5 & $31(35,2)$ & $24(27,3)$ & $33(33,5)$ & $63(34,1)$ & $28(15,2)$ & $94(50,8)$ \\
\hline \multirow[t]{2}{*}{5 e mais } & $68(46,3)$ & $25(17,0)$ & $54(36,7)$ & $58(30,9)$ & $30(16,0)$ & $100(53,2)$ \\
\hline & \multicolumn{3}{|c|}{ Valor de $\mathrm{p}<0,000$} & \multicolumn{3}{|c|}{ Valor de $\mathrm{p}<0,000$} \\
\hline \multicolumn{7}{|l|}{ HORAS TRABALHADAS } \\
\hline Até 14 horas & $5(4,1)$ & $18(14,9)$ & $98(81,0)$ & $25(4,5)$ & $56(10,1)$ & $473(85,4)$ \\
\hline 15 a 39 horas & $52(12,2)$ & $46(10,7)$ & $330(77,1)$ & $101(6,1)$ & $211(12,7)$ & $1350(81,2)$ \\
\hline \multirow[t]{2}{*}{40 e mais } & $142(22,3)$ & $88(13,8)$ & $408(64,0)$ & $247(10,4)$ & $368(15,5)$ & $1752(74,0)$ \\
\hline & \multicolumn{3}{|c|}{ Valor de $p<0,000$} & \multicolumn{3}{|c|}{ Valor de $p<0,000$} \\
\hline
\end{tabular}

Fonte: PNAD/IBGE - Pesquisa Nacional de Amostra por domicílios, 2008.

Nota: ${ }^{*}$ com e sem carteira assinada; ${ }^{* *}$ conta própria, empregadora, trabalhadora para o próprio consumo, ${ }^{* * *}$ salário mínimo de referência R\$ 415,00. 


\section{Resultados}

As Tabelas 1 e 2 apresentam distribuição proporcional das características sócio-demográficas e condições de trabalho, estratificadas por raça/cor, bem como os níveis de acesso aos serviços preventivos de saúde. Sobre o acesso em relação à raça/cor, observou-se, na Tabela 1, que, para o acesso considerado bom, as mulheres brancas representam $15,4 \%$, enquanto que as mulheres negras, 7,9\% do total; para o acesso regular, o indicador é representado por mais de $10 \%$ das mulheres $(11,8 \%$ brancas e $13,6 \%$ negras). No entanto, no que se refere ao acesso ruim, as mulheres apresentam uma alta concentração, chegando a mais de $70 \%$ do total $(72,8 \%$ brancas e $78,6 \%$ negras). Em ambos os grupos (18,8\% brancas e $10,4 \%$ negras), a relação entre o acesso e a distribuição etária mostra que há uma tendência de aumento do acesso com o aumento do grupo etário. Entretanto, as mulheres de 25 a 34 anos $(80,4 \%$ brancas e $86,5 \%$ negras) têm um maior percentual do nível de acesso considerado ruim (Tabela 1 ).

$\mathrm{Na}$ composição familiar e no acesso aos serviços de saúde, as mulheres que fazem parte do grupo "outros arranjos familiares" apresentam a maior proporção do acesso considerado bom para ambos os grupos de mulheres (18,1\% brancas e 9,8\% negras). Na categoria de acesso ruim, as mães solteiras $(75,4 \%$ brancas e $79,2 \%$ negras) apresentam o maior percentual (Tabela 1).

$\mathrm{Na}$ relação entre acesso, raça/cor e níveis de instruçáo, os dados revelam redução do acesso entre as mulheres com menores níveis de instrução, pois as mulheres sem instrução $(90,6 \%$ das mulheres negras e $92,8 \%$ das mulheres brancas) não acessam o serviço. Em ambos os grupos, a melhora do acesso ocorre a partir dos níveis médio (20,5\% brancas e $12,4 \%$ negras) e superior (41,6\% brancas e $17,6 \%$ negras), como demonstra a Tabela 1.

A relação entre acesso e posição das mulheres no mercado de trabalho mostra expressivas variações entre os diferentes níveis de ocupação, em ambos os grupos. Nos resultados apresentados, é possível perceber que as mulheres que possuem carteira assinada $(27,9 \%$ brancas e $15 \%$ negras) ou são funcionárias públicas (34,6\% brancas e $27,2 \%$ negras) foram contempladas com o bom acesso, que se concentrou em maior proporção nessas categorias, conforme Tabela 2. Já entre as trabalhadoras, sejam negras ou brancas, as mulheres que não têm acesso são as: sem carteira assinada (79,7\% brancas e $82,6 \%$ negras), trabalhadoras domésticas $(88,7 \%$ brancas e $83,6 \%$ negras) e não remuneradas (87,6\% brancas e 93,3\% negras). Com relação à condição da atividade, a distribuição é semelhante em ambos os grupos de mulheres, sendo as mulheres economicamente ativas (15,8\% brancas e 7,7\% negras) e não economicamente ativas ( $14,7 \%$ brancas e $8,3 \%$ negras) responsáveis pelos maiores percentuais no acesso ruim (Tabela 2 ).

A relação entre os níveis de renda e o acesso aos serviços de saúde é direta. Quanto menor a renda menor o acesso, como pode ser observado na Tabela 2. Em ambos os grupos, nota-se uma diminuição no nível do acesso entre aquelas que recebem menos do que um salário mínimo (86,2\% brancas e 87,3\% negras). Dentre as brancas, quanto maior o nível de renda, maior o acesso aos serviços de saúde. Dentre as negras, há aumento do acesso entre um e três salários mínimos e redução nos demais níveis de renda. A relação entre horas trabalhadas e acesso é semelhante ao padrão apresentado pela renda pessoal, ou seja, quanto maior o número de horas trabalhadas maior o acesso aos serviços de saúde em ambos os grupos.

\section{Conclusão}

Os níveis de instrução e renda pessoal são considerados macrodeterminantes, sendo caracterizados pelas condiçóes econômicas, culturais e ambientais de uma sociedade. No estudo, podem-se identificar as características sociais e demográficas como influenciadoras do acesso das mulheres aos exames preventivos de saúde, pois, quanto melhor a renda, nível de instrução e ocupação no mercado de trabalho melhor o acesso.

Fatores associados às desigualdades de gênero e raça determinam as disparidades sociais, hierarquizando o acesso aos serviços de saúde por meio das diferentes características individuais. Entretanto, apesar de as mulheres negras e brancas sofrerem o impacto das 
desigualdades sociais, o racismo determina as condiçóes de acesso das mulheres negras, como se pode depreender dos dados; para o acesso ruim, as mulheres negras têm o maior percentual, enquanto as mulheres brancas são prioridade no acesso bom.

O fator idade também é determinante para o acesso aos exames preventivos de saúde, pois o estudo demonstrou que quanto maior a idade melhor o acesso. No entanto, ao se analisar por raça/cor, as mulheres negras com 50 anos ou mais têm o acesso prejudicado quando comparadas com as mulheres brancas.

De acordo com Bairros, Meneghel e Olinto (2008), as mulheres negras mais velhas tornam-se ainda mais vulneráveis socialmente, pois, com mais de 40 anos de idade, elas têm maior probabilidade de não ter realizado exame citopatológico ou de estar com o exame em atraso. A discriminação geracional impacta o acesso das mulheres mais velhas aos serviços de saúde.

As mulheres com nível de instrução mais elevado têm melhor acesso aos serviços preventivos de saúde, numa relação direta. Neste caso, as mulheres brancas têm o maior percentual quando comparadas às mulheres negras.

As dificuldades encontradas por pretos e pardos no sistema educacional do Estado de São Paulo não diferem das encontradas em outras partes do Brasil, sendo possível estabelecer uma correlação entre a cor e o analfabetismo, repetência, evasão escolar e condiçóes de vida, sempre com piores resultados para os pretos (BATISTA, 2002).

Um estudo realizado com as PNADs de 1998 e 2003 demonstrou que as pessoas de maior renda e escolaridade apresentaram maior probabilidade de ter realizado consulta quando comparadas com as pessoas sem escolaridade e renda mais baixa. Quanto mais alta a renda e a escolaridade maior será a probabilidade de o indivíduo ter consultado médico nos últimos doze meses (MOTTA; FAHEL; PIMENTEL, 2008).

Os níveis de instrução e renda são aspectos sociais e econômicos determinantes das condiçóes de vida de uma população, que podem, inversamente, caracterizar uma sociedade estruturada pelas desigualdades raciais onde as mulheres negras tendem a estar em maior exposição sob o aspecto do acesso desqualificado aos serviços de saúde.

Em relação à posição no mercado de trabalho, o estudo evidenciou que as mulheres que são funcionárias públicas têm melhor acesso aos serviços preventivos de saúde. Isso pode ser explicado pelo fato de essas mulheres terem acesso à cobertura de plano de saúde que garanta o acesso aos serviços.

Para Rodrigues (2009), que realizou um estudo em Salvador, ficou demonstrado que as mulheres usuárias de serviços públicos de pré-natal e parto, negras em sua maioria, possuem, em grande parte, baixos indicadores sociais e econômicos, já que a maioria possui renda de até dois salários mínimos, exerce atividade sem remuneração e tem, em média, 9,62 anos de estudo.

Um estudo sobre acesso aos exames de detecção precoce para saúde da mulher na Região Sul do Brasil observou, quanto às características sociais e econômicas, que $56 \%$ das mulheres negras apresentaram menos do que oito anos de escolaridade; $45 \%$ pertenciam às classes D e E; e $89 \%$ viviam com renda familiar per capita menor ou igual a três salários mínimos (BAIRROS; MENEGHEL; OLINTO, 2008).

De acordo com Leal, Gama e Cunha (2005), as mulheres também se diferenciam nos serviços de saúde segundo o grau de instrução e a cor da pele no pré-natal. As mulheres negras com menor nível de instrução tiveram menor acesso à atenção adequada, de acordo aos padróes estabelecidos pelo Ministério da Saúde. Dessa forma, não há como deixar de constatar que na sociedade há dois níveis de discriminação: a educacional e a racial, que invadem a esfera da atenção oferecida pelos serviços de saúde.

Lopes, Buchalla e Ayres (2007), em seu estudo sobre Mulheres negras e não negras e vulnerabilidades ao HIVIAIDS no Estado de São Paulo, informam que as mulheres negras entrevistadas eram detentoras do pior status sócio-econômico, seja na escolaridade, no rendimento ou nas condiçôes de moradia.

Batista (2002) alega que o menor acesso aos bens e ao consumo social, menor escolaridade e dificuldade de inserção da população negra no mercado de trabalho ajudam a entender por que a população negra possui os piores indicadores de mortalidade, principalmente 
quando as causas ou os fatores diretamente ligados à morte têm relação com as condições de vida, acesso aos serviços de saúde e qualidade da atenção.

Ao agregar as desigualdades sócio-demográficas e raciais, as mulheres negras ficam mais expostas às iniquidades em saúde, o que causa prejuízo ao processo saúde-doença, impactando as condições de vida. As condições sociais e demográficas são determinantes para o acesso aos serviços preventivos de saúde. No entanto, para as mulheres negras, a situação é sempre desfavorável, mesmo quando se equiparam às mulheres brancas quanto às melhores rendas, níveis de instrução e ocupação no mercado de trabalho.

As desigualdades raciais determinam o acesso aos serviços de saúde e limitam o cuidado. Por intermédio do racismo, as desigualdades são causadoras de doenças e agravos que resultam nas iniquidades raciais em saúde. E, para as mulheres negras, outros fatores agregados, como o sexismo, expóem a uma situação de vulnerabilidade e violam o direito à saúde e ao acesso qualificado.

\section{Discussão}

Os determinantes sociais influenciam o acesso das mulheres aos serviços preventivos de saúde. $\mathrm{O}$ estudo demonstrou que mesmo as mulheres com melhor renda, nível de instrução e ocupação no mercado de trabalho, em alguma medida, são prejudicadas pela falta de acesso a esses exames, podendo-se afirmar que as políticas de atenção à saúde das mulheres não estão sendo suficientemente implementadas nos serviços de saúde.

Apesar do Sistema Público de Saúde (SUS) ser universal, igualitário e equânime, com garantia constitucional conferida a todas (os) as (os) cidadãs (os) brasileiras (os), independentemente de sua cor/raça, sexo ou qualquer outra característica, sua aplicabilidade ideal ainda está distante, devido a fatores de contexto histórico, cultural e político.

O estudo apresentou o racismo institucional como uma barreira ao acesso aos serviços preventivos para a saúde das mulheres negras. E que essas desigualdades são determinantes sociais que impactam as condições de vida e o processo de adoecimento.

\section{Referências}

BAIRROS, F.S.; MENEGHEL, S.N.; OLINTO, M.T.A. Citopatológico e exame de mama: desigualdade de acesso para mulheres negras no sul do Brasil. Epidemiologia e Serviços de Saúde, Brasília, 17(2):123-153, abr-jun, 2008.

BARATA, R.B. Como e por que as desigualdades sociais fazem mal à saúde. Rio de Janeiro: FIOCRUZ, 2009.

Condições de vida e situação de saúde. Rio de Janeiro. ABRASCO, 1997

BATISTA, L.E. Mulheres e homens negros: saúde, doença e morte. 2002. Tese (Doutorado em Sociologia) - Universidade Estadual Paulista, Araraquara, 2002.

BATISTA, L.E; ESCUDER, M.M.L. Medindo desigualdade na saúde. Boletim Epidemiológico Paulista. abr., ano 2, n. 16, 2005.

CARNEIRO, S. "A batalha de Durban". Revista Estudos Feministas, Florianópolis, v. 10, n. 1, p. 2.009-2.014, 2002.
CRENSHAW, K. Documento para o encontro de especialistas em aspectos da discriminação racial relativos ao gênero. Revista Estudos Feministas, v.10, n.1, p.171-188, 2002.

CRI. Articulação para o Combate ao Racismo Institucional. Identificação e abordagem do racismo institucional. Brasília: CRI, 2006.

HERINGER, R. Desigualdades raciais no Brasil: síntese de indicadores e desafios no campo das políticas públicas. Cadernos de Saúde Pública [online]. 2002, v. 18 supl., p. 57-65. Disponível em: <http:// www.scielo.br/scielo>. Acesso em: 27 de abril de 2010.

IANNI, O. Dialética das relações raciais. Estudos avançados, São Paulo, v. 18, n.50, abr. 2004. Disponível em: <http://www.scielo. br/scielo>. Acessos em 04 jul. 2010.

LEAL, M.C.; GAMA, S.G.N.; CUNHA, C.B. Desigualdades raciais, sócio-demográficas e na assistência ao pré-natal e ao parto, 19992001. Revista de Saúde Pública. São Paulo, v. 39, n. 1, 2005. 
LOPES, F.; BUCHALLA, C.M.; AYRES, J.R.C.M. Mulheres negras e nãonegras e vulnerabilidade ao HIV/Aids no estado de São Paulo, Brasil. Revista de Saúde Pública, v. 41, supl. 2, p. 39-46, 2007.

MOTTA, C.P.G.; FAHEL, M.C.X.; PIMENTEL, E.C. Desigualdades no acesso aos serviços de saúde da população ocupada: uma análise a partir dos dados das PNADS 1998 e 2003. Trabalho apresentado no XVI Encontro Nacional de Estudos Populacionais, ABEP, realizado em Caxambu, MG - Brasil, de 29 de setembro a 03 de outubro de 2008 .
RODRIGUES, Q.P. Desigualdades raciais no acesso de mulheres ao cuidado pré-natal e no parto. 2009. 98f. Dissertação (Mestrado em Enfermagem) - Escola de Enfermagem, Universidade Federal da Bahia, 2009.

Recebido para publicação em novembro de 2013

Versão final em dezembro de 2013

Conflito de interesse: não houve

suporte financeiro: inexistente 